BNL-111772-2015-IR

File \# 92688

\title{
Defining the safe current limit for opening ID photon shutter
}

\author{
S. Seletskiy
}

December 2015

Photon Sciences Department

Brookhaven National Laboratory

\author{
U.S. Department of Energy \\ USDOE Office of Science (SC), \\ Basic Energy Sciences (BES) (SC-22)
}

Notice: This manuscript has been authored by employees of Brookhaven Science Associates, LLC under Contract No. DE- SC0012704 with the U.S. Department of Energy. The publisher by accepting the manuscript for publication acknowledges that the United States Government retains a non-exclusive, paid-up, irrevocable, world-wide license to publish or reproduce the published form of this manuscript, or allow others to do so, for United States Government purposes. 


\section{DISCLAIMER}

This report was prepared as an account of work sponsored by an agency of the United States Government. Neither the United States Government nor any agency thereof, nor any of their employees, nor any of their contractors, subcontractors, or their employees, makes any warranty, express or implied, or assumes any legal liability or responsibility for the accuracy, completeness, or any third party's use or the results of such use of any information, apparatus, product, or process disclosed, or represents that its use would not infringe privately owned rights. Reference herein to any specific commercial product, process, or service by trade name, trademark, manufacturer, or otherwise, does not necessarily constitute or imply its endorsement, recommendation, or favoring by the United States Government or any agency thereof or its contractors or subcontractors. The views and opinions of authors expressed herein do not necessarily state or reflect those of the United States Government or any agency thereof. 
BNL-111772-2015-IR

\title{
Defining the safe current limit for opening ID photon shutter
}

\author{
S. Seletskiy
}

$12 / 14 / 15$

\section{Introduction}

The NSLS-II storage ring is protected from possible damage from insertion devices (IDs) synchrotron radiation by a dedicated active interlock system (AIS) [1]. It monitors electron beam position and angle and triggers beam drop if beam orbit exceeds the boundaries of precalculated active interlock envelope (AIE) [2].

The beamlines $(\mathrm{BL})$ and beamline frontends (FE) are designed under assumption that the electron beam is interlocked within the AIE. For historic reasons the AIS engages the ID active interlock (AI-ID) at any non-zero beam current whenever the ID photon shutter (IDPS) is getting opened. Such arrangement creates major inconveniences for BLs commissioning.

Apparently there is some IDPS safe current limit (SCL) under which the IDPS can be opened without interlocking the e-beam. The goal of this paper is to find such limit.

\section{Current AIS setup}

The most recent AIS setup is schematically shown in Fig. 1. The AI-ID is getting engaged for open IDPS at beam current of $0.2 \mathrm{~mA}$ (the lowest reliably readable non-zero current).

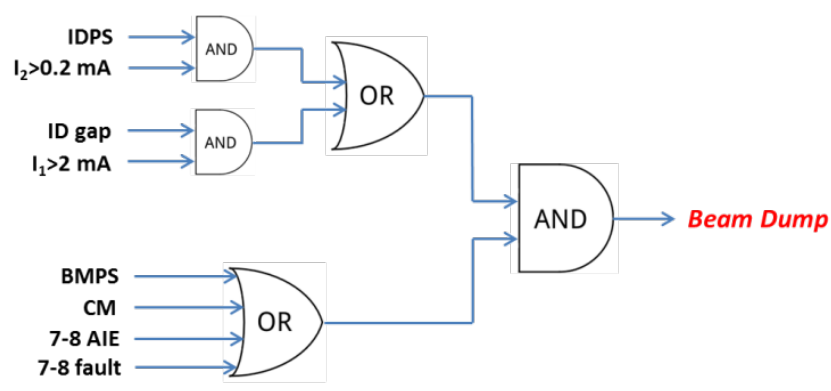

\begin{tabular}{|l|c|c|}
\hline & 0 & 1 \\
\hline IDPS & closed & open \\
\hline $\mathrm{I}_{1}$ & $<2 \mathrm{~mA}$ & $>=2 \mathrm{~mA}$ \\
\hline $\mathrm{I}_{2}$ & $<0.2 \mathrm{~mA}$ & $>=0.2 \mathrm{~mA}$ \\
\hline ID (gap) & gap open & gap closed \\
\hline $\begin{array}{l}\text { BPM } \\
\text { (positon/angle) }\end{array}$ & $\begin{array}{c}\text { all within Al } \\
\text { limits }\end{array}$ & $\begin{array}{c}\text { some out of } \\
\text { Al limits }\end{array}$ \\
\hline CM (current) & within range & out of range \\
\hline BMPS & open & close \\
\hline
\end{tabular}

Figure 1: Schematics of the most recent AI-ID setup.

It was determined [3] that with the IDPS closed the FEs can be operated safely with not interlocked e-beam under $2 \mathrm{~mA}$ beam current. Below we will define the SCL below which both 
the typical FE and the typical BL can be safely operated with open IDPS and not interlocked ebeam.

\section{Finding IDSP SCL from comparing ID and BM synchrotron radiation}

Any NSLS-II frontend and beamline shall be able to accept the power from the bending magnet (BM) synchrotron radiation at $0.5 \mathrm{~A}$ current with electron beam interlocked horizontally within $+/-5 \mathrm{~mm}$ and vertically within $+/-3 \mathrm{~mm}$ at the locations of the 180 regular BPMs. That is at any beam current the incident angle of synchrotron radiation fan (SRF) from the BM on the $\mathrm{FE} / \mathrm{BL}$ in-vacuum components downstream of the FE fixed mask (FM) is defined by the FE/BL geometry rather than the e-beam interlock.

The angular power density of the BM SRF in horizontal direction is close to Heaviside step function and in vertical direction is defined by [4]:

$$
\frac{d^{2} P}{d \psi d \theta}=\frac{21 \gamma C_{\gamma} E^{4} I \cdot 10^{9}}{64 \pi \rho\left(1+(\gamma \theta)^{2}\right)^{\frac{5}{2}}}\left(1+\frac{5}{7} \cdot \frac{(\gamma \theta)^{2}}{1+(\gamma \theta)^{2}}\right)
$$

Here $\gamma$ is the relativistic factor, $C_{\gamma}=8.846 \cdot 10^{-5} \mathrm{~m} / \mathrm{GeV}$, beam energy $E=3 \mathrm{GeV}$, and $\mathrm{BM}$ radius $\rho=25 \mathrm{~m}$.

The angular power density distribution of a planar undulator SRF $[2,5]$ is given by:

$$
\begin{aligned}
& \frac{d^{2} P}{d \psi d \theta}=\frac{6 P_{T} \gamma^{2}}{(\pi K)^{2}} \cdot \int_{b-K}^{b+K} \sqrt{K-(b-y)^{2}} \frac{a^{2}+(2 a-4) y^{2}+y^{4}}{\left(a+y^{2}\right)^{5}} d y \\
& a=1+(\gamma \psi)^{2}, b=\gamma \theta \\
& P_{T}=0.633 L I E^{2} B^{2}
\end{aligned}
$$

Here $L$ is the undulator length and $B$ is magnetic field of the undulator.

It is obvious from above considerations that the FE/BL component at some distance from the ID SRF source can accommodate the ID SRF power at some beam current with not interlocked e-beam as long as the peak areal power density of the ID SRF at this beam current is smaller than the peak areal power density of the BM SRF at beam current of $0.5 \mathrm{~A}$.

For any beamline the SRF power is of concern down to the location of the monochromator [6]. The typical monochromator is located $30 \mathrm{~m}$ downstream of the center of the ID [7]. Thus, to find the IDPS SCL it is enough to compare areal power densities of the BM and ID SRF from BMFE interface (BMA exit) to the monochromator.

For the sake of integrity we will compare the BM SRF to the synchrotron radiation from two most extreme IDs. One ID is the damping wiggler (DW) producing the highest total power ( $32 \mathrm{x}$ $2 \mathrm{~kW}$ per cell at $0.5 \mathrm{~A}$ current) and having the widest horizontal synchrotron radiation fan. Another ID is the inelastic X-Ray scattering undulator (IXS), which produces the SRF with the 
highest peak angular power density. The respective $K, B$ and $L$ values for these IDs are given in [8].

Both DW and IXS are located in the long straight sections of the storage ring. The distance from the center of the long straight section to the BMA exit is $11.44 \mathrm{~m}$. The distance from the source of the $B M$ radiation to the BMA exit can be taken to be $2.5 \mathrm{~m}$.

We find peak angular power of ID and BM SRF from (1) and (2) and calculate the peak areal power density at distance $z$ downstream of BMA exit as:

$$
\frac{d^{2} P}{d x d y}=\frac{d^{2} P}{d \psi d \theta} \cdot \frac{1}{\left(z+z_{0}\right)^{2}}
$$

where $z_{0}$ is the distance from SRF source to the BMA exit. Figure 2 shows the areal peak power of the BM synchrotron radiation fan at $0.5 \mathrm{~A}$ and the areal peak powers of the DW and the IXS $\mathrm{SRF}$ at $1 \mathrm{~mA}$ from BMA exit to the location of the BL monochromator.

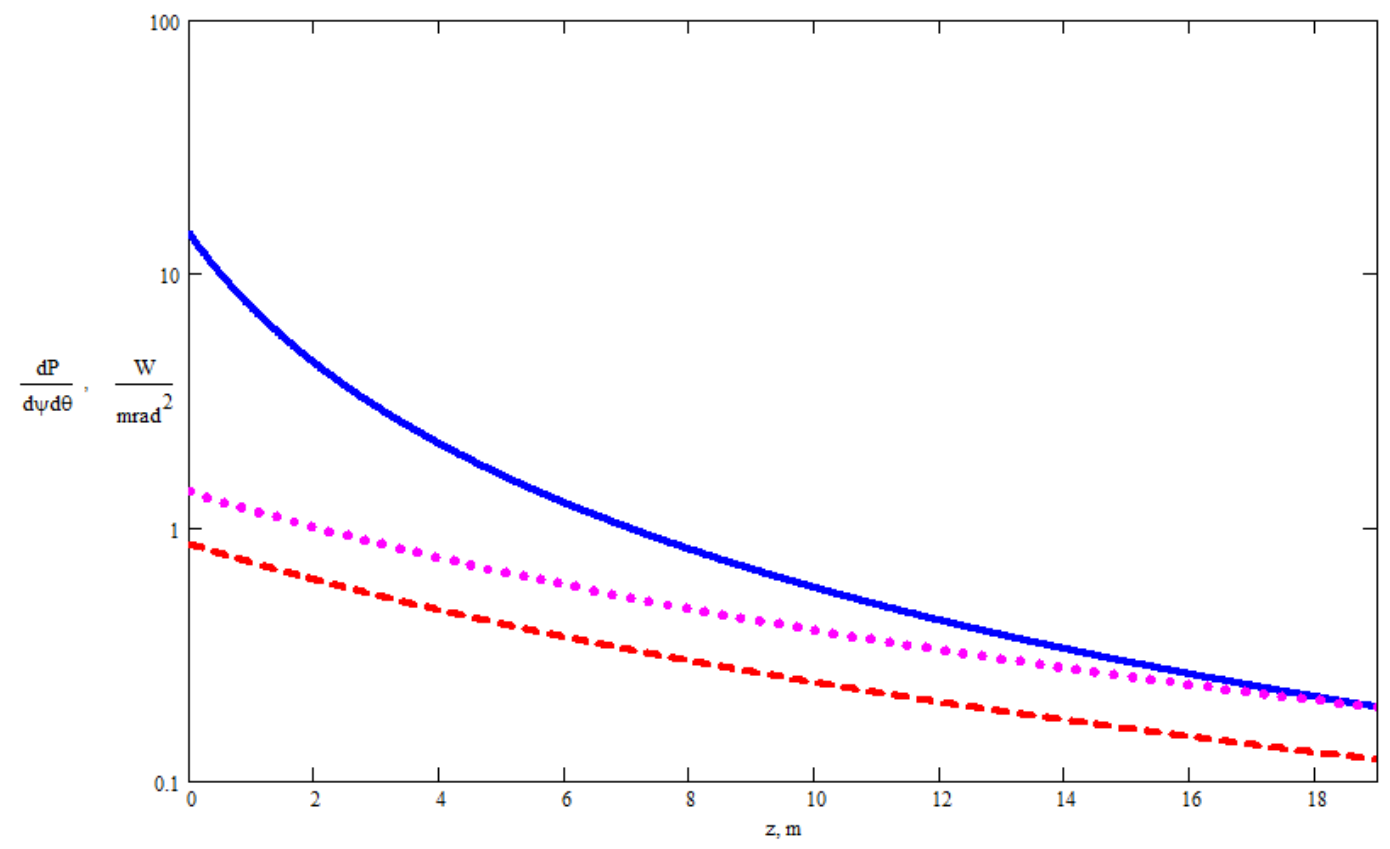

Figure 2: Areal peak power of the BM synchrotron radiation fan at $0.5 \mathrm{~A}$ (blue) and areal peak powers of the DW (red) and the IXS (magenta) SRF at $1 \mathrm{~mA}$. The BMA exit is located at $0 \mathrm{~m}$, the monochromator is located approximately at $18.6 \mathrm{~m}$.

The above considerations show that it is absolutely safe to set the IDPS SCL to $1 \mathrm{~mA}$. 


\section{Finding IDSP SCL from comparing ID synchrotron radiation from interlocked and not interlocked e-beam}

Any NSLS-II frontend and beamline shall be able to accept the power from the ID synchrotron radiation at $0.5 \mathrm{~A}$ current with electron beam interlocked at the center of the ID both horizontally and vertically within $+/-0.25 \mathrm{mrad}$ and $+/-0.5 \mathrm{~mm}$.

If at some current the e-beam is not interlocked than downstream of the FM the angle of incident of the ID SRF on the FE/BL component is defined by the transverse displacement of the e-beam at the SRF source, the FM half-aperture $\left(a_{F M}\right)$ and the distance $(D)$ from ID center to the FM. Since FM has larger horizontal aperture and ID SRF is wider in horizontal direction it is enough to consider horizontal beam displacements $(x)$ only.

Under extreme scenario with not interlocked e-beam the in-vacuum surface will see the peak ID SRF power density $\left.\frac{d^{2} P}{d \psi d \theta}\right|_{\psi=0, \theta=0}$ at incident angle $\frac{a_{F M}+x}{D}$. Under this scenario with interlocked beam the same in-vacuum surface will see ID SRF power density of $\left.\frac{d^{2} P}{d \psi d \theta}\right|_{\psi=\left(a_{F M}+x\right)-0.25 \mathrm{mrad}, \theta=0}$ at incident angle $0.25 \mathrm{mrad}$. Thus, the IDPS SCL $\left(I_{2}\right)$ is defined by the following equation:

$$
\left.\frac{a_{F M}+x}{D} \cdot \frac{d^{2} P\left(I_{2}\right)}{d \psi d \theta}\right|_{\psi=0, \theta=0}=\left.0.25 \cdot \frac{d^{2} P(0.5 A)}{d \psi d \theta}\right|_{\psi=\left(a_{F M}+x\right)-0.25, \theta=0}
$$

Substituting (2) into (3) for the IXS and for the DW and taking into account that FM halfapertures are $5 \mathrm{~mm}$ and $10 \mathrm{~mm}$ for IXS and DW respectively we obtain $I_{2}(x)$ dependences demonstrated in Fig. 3.
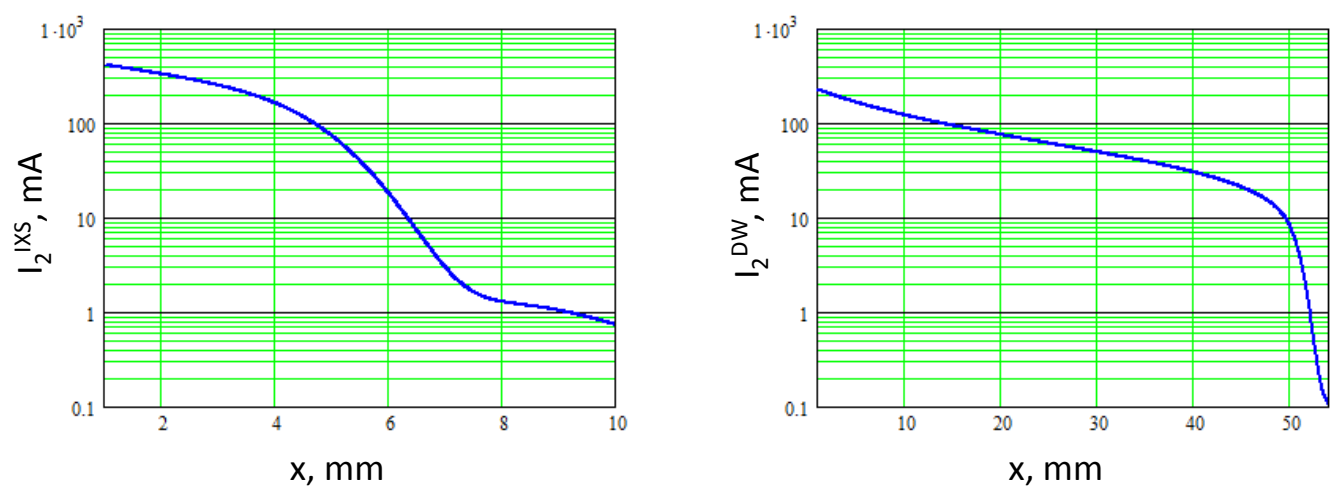

Figure 3: Dependence of IDPS SCL on the possible horizontal displacement of e-beam at the center of the ID straight for the IXS (left plot) and DW (right plot) insertion devices. 
As one can see, the above considerations show that $2 \mathrm{~mA}$ IDPS SCL is indeed $100 \%$ safe for the DW since $51.6 \mathrm{~mm}$ displacement corresponding to such $\mathrm{SCL}$ is larger than the physical aperture of the straight section vacuum chamber. For IXS the $2 \mathrm{~mA}$ limit is reached at beam displacement of $7.3 \mathrm{~mm}$, which is also extremely large and for practical reasons is never reached in beam studies. Here we are discussing the e-beam closed orbit that is displaced at the center of the long straight section by $7.3 \mathrm{~mm}$ and inclined by $0.625 \mathrm{mrad}$, which gives more than $10 \mathrm{~mm}$ displacement at the neighboring quadrupole.

\section{We conclude that the reasonable IDPS SCL is $2 \mathrm{~mA}$.}

\section{Conclusion}

In this paper we found the practical safe current limit for opening the insertion device photon shutter. In our analysis we relied on the fact that by design any NSLS-II frontend or beamline must accept the power load from the synchrotron radiation produced by virtually not interlocked e-beam in the bending magnet and by interlocked beam in respective ID at full beam current. By comparing power density from such synchrotron radiation fans to the power density produced by not interlocked electron beam in two most powerful IDs (damping wiggler and IXS undulator) we conclude that the reasonable practical IDPS safe current limit is $\mathbf{2} \mathbf{m A}$.

\section{References}

[1] S. Seletskiy et al., Commissioning of Active Interlock System for NSLS-II Storage Ring, TUPMA057, IPAC15 Proceedings, 2015.

[2] S. Seletskiy, T. Shaftan, Reviewed approach to defining the Active Interlock Envelope for Front End ray tracing, BNL-108464-2015-IR, 2015.

[3] S. Seletskiy, C. Amundsen, K. Ha, A. Hussain, Safe operating conditions for NSLS-II Storage Ring Frontends commissioning, BNL-107813-2015-IR, 2015.

[4] A. W. Chao, M. Tigner (editors), Accelerator Physics and Engineering, ISBN 9810238584, 1999.

[5] K. J. Kim, Nuclear Instruments and Methods A246 (1986) 67-70.

[6] Private communications with A. Suvorov.

[7] NSLS-II CDR, 2006.

[8] A. Broadbent, RSI for the Front-Ends for the Six NSLS-II Project Beamlines, 2011. 